\title{
Stoichiometric network analysis of a reaction system with conservation constraints
}

\author{
Željko Čupić, ${ }^{1}$ Stevan Maćešić, ${ }^{2, a)}$ Katarina Novakovic, ${ }^{3}$ Slobodan Anić, ${ }^{1,2}$ and Ljiljana Kolar-Anić ${ }^{2}$ \\ ${ }^{1}$ Centre of Catalysis and Chemical Engineering, Institute of Chemistry, Technology and Metallurgy, University of Belgrade, \\ Njegoševa 12, 11000 Belgrade, Serbia \\ ${ }^{2}$ Faculty of Physical Chemistry, University of Belgrade, Studentski trg 12, 11000 Belgrade, Serbia \\ ${ }^{3}$ School of Engineering, Newcastle University, Merz Court, Newcastle upon Tyne NE1 7RU, United Kingdom
}

(Received 23 February 2018; accepted 26 July 2018; published online 17 August 2018)

\begin{abstract}
Stoichiometric Network Analysis (SNA) is a powerful method that can be used to examine instabilities in modelling a broad range of reaction systems without knowing the explicit values of reaction rate constants. Due to a lack of understanding, SNA is rarely used and its full potential is not yet fulfilled. Using the oscillatory carbonylation of a polymeric substrate [poly(ethylene glycol)methyl ether acetylene] as a case study, in this work, we consider two mathematical methods for the application of SNA to the reaction models when conservation constraints between species have an important role. The first method takes conservation constraints into account and uses only independent intermediate species, while the second method applies to the full set of intermediate species, without the separation of independent and dependent variables. Both methods are used for examination of steady state stability by means of a characteristic polynomial and related Jacobian matrix. It was shown that both methods give the same results. Therefore, as the second method is simpler, we suggest it as a more straightforward method for the applications. Published by AIP Publishing. https://doi. org/10.1063/1.5026791
\end{abstract}

\section{NOMENCLATURE}

\begin{tabular}{|c|c|c|}
\hline $\mathrm{CH}_{3} \mathrm{OH}$ & $=$ & methanol \\
\hline $\mathrm{CO}$ & $=$ & carbon monoxide \\
\hline HI & $=$ & hydroiodic acid \\
\hline $\mathrm{I}_{2}$ & $=$ & iodine \\
\hline IPdR & $=$ & (methoxycarbonyl)palladium(II) iodide \\
\hline $\mathrm{O}_{2}$ & $=$ & oxygen \\
\hline $\mathrm{Pd}$ & $=$ & palladium \\
\hline $\mathrm{PdI}_{2}$ & $=$ & palladium iodide \\
\hline PEGA & $=$ & poly(ethylene glycol)methyl ether acetylene \\
\hline PEGP & $=$ & $\begin{array}{l}\text { poly(ethylene glycol)methyl ether }(\mathrm{Z})-5- \\
\text { methoxy-3-(methoxycarbonyl)-5-oxopent-3- } \\
\text { enoate; ester product }\end{array}$ \\
\hline
\end{tabular}

Oscillating chemical reactions are fascinating chemical systems characterized by their self-organizing capabilities. The complexity of the systems' dynamic states makes it a challenge to understand and model the processes. Sophisticated methods are required, and among them stoichiometric network analysis (SNA) is a powerful technique. In oscillating chemical reactions, as in all chemical systems, certain mass conservation constraints among the chemical species apply. These mass conservation constraints dividing all intermediate species into the independent and dependent ones can have a great impact on the stability of the oscillating chemical reactions, but insufficient attention has been given to their role. In this paper, we consider two different mathematical approaches, both based on SNA, that address this problem, utilise mass conservation constraints, and determine instability regions. As

\footnotetext{
a) Author to whom correspondence should be addressed:
} stevan.macesic@ffh.bg.ac.rs an example, we considered the oscillatory carbonylation of poly(ethylene glycol)methyl ether acetylene (PEGA), where two constraints, representing the conservation of palladium (Pd) and iodine (I) atoms, served in the model to express the concentrations of two dependant species as a function of the remaining independent ones. The two aforementioned SNA mathematical approaches were shown to give the same result. Based on these, we are able to suggest the method more appropriate for the application. Thus, using the simpler method, we can more quickly and more easily obtain the instability region where oscillatory dynamics emerges which is important for the application of oscillatory reactions, particularly in biology and industry. This is important since in the case considered here, as in many other complex reactions, the relative concentrations of reaction products often depend on dynamic states of a reaction system. Biological systems are always in oscillatory dynamic states; any deviance from those states is related to illness. Hence, modelling of the reaction system together with the prediction of its oscillatory dynamics is very important, but, long and complicated calculations are not practical and many scientists give up on that task.

\section{INTRODUCTION}

When a nonlinear system is under nonequilibrium conditions, its basic steady state can be unstable in the well defined region of initial conditions. An excellent method to examine the steady state stability in models of chemical, physicochemical, and biochemical reaction systems is stoichiometric network analysis (SNA). ${ }^{1,2}$ This method can 
be successfully applied to multi-variable models where the variables are the concentrations of intermediate species. In the case when the conservation constraints between these species have an important role, SNA requires additional mathematical manipulations. In reality, the conservation constraints between species always exist in the reaction systems. The common way to avoid this problem is to assume that some of the species mentioned are in excess, and therefore, their concentrations may be considered constant. However, if this assumption is not valid, we need to apply one of two mathematical methods briefly mentioned by Bruce Clark in one of his publications (pp. 46-47 in Ref. 1). In this work, we present methods reinforced by in depth explanations, aiming to release the full power of these approaches, and make them accessible to the broader scientific community. The palladium-catalysed oscillatory carbonylation of mono-alkyne-terminated poly(ethylene glycol)methyl ether acetylene (PEGA), hereafter referred to as the oscillatory carbonylation of PEGA (Sec. II), ${ }^{3-5}$ is used as the model system for the comprehensive illustration of these two procedures. The first method (Sec. III A) applies to the independent intermediate species only and takes conservation constraints into account for examination of steady state stability, while the second method (Sec. III B) applies to the full set of intermediate species, without the separation of independent and dependent variables.

SNA of the reaction system with thermodynamic constraints was treated elsewhere. ${ }^{6}$

\section{MODEL SYSTEM: PALLADIUM-CATALYSED OSCILLATORY CARBONYLATION OF PEGA}

Oscillatory carbonylation reactions have been researched in the past twenty years with the majority of studies being experimental. ${ }^{3,7-13}$ Polymeric substrate oscillatory carbonylation is a recent addition to the family of oscillatory carbonylation reactions. The interest in instability of reactions with polymeric substrates is increasing with recent achievements in control of front polymerization by autocatalytic processes. $^{14-16}$

During experimental investigations of the palladiumcatalysed carbonylation of PEGA, the oscillatory dynamic states of this reaction system are found and simulated numerically by differential equations based on the simplified reaction mechanism given in Table I.

Time evolution of this reaction network is described by a system of differential equations:

$$
\left[\begin{array}{l}
\mathrm{d} c_{1} / \mathrm{d} t \\
\mathrm{~d} c_{2} / \mathrm{d} t \\
\mathrm{~d} c_{3} / \mathrm{d} t \\
\mathrm{~d} c_{4} / \mathrm{d} t \\
\mathrm{~d} c_{5} / \mathrm{d} t \\
\mathrm{~d} c_{6} / \mathrm{d} t
\end{array}\right]=\left[\begin{array}{cccccc}
-1 & 0 & 1 & 1 & -1 & 1 \\
1 & 0 & -1 & -1 & 0 & 0 \\
2 & -2 & 0 & 0 & 1 & -1 \\
0 & -1 & -1 & -1 & 0 & 0 \\
0 & 0 & 0 & 0 & 1 & -1 \\
-1 & 0 & 0 & 0 & 0 & 0
\end{array}\right]\left[\begin{array}{l}
r_{1} \\
r_{2} \\
r_{3} \\
r_{4} \\
r_{5} \\
r_{6}
\end{array}\right] .
$$

Here, $\mathrm{d} c_{j} / \mathrm{d} t$ is the time derivative of the concentration of species $j$.

Equation (1) can be also written in the matrix form

$$
\frac{\mathrm{d} c}{\mathrm{~d} t}=\mathbf{S} \boldsymbol{r},
$$

TABLE I. Oscillatory carbonylation of PEGA (see Nomenclature for all nonconventional assignations). The reaction network consists of chemical reactions adapted to fit in the rate equations $r_{i}$ ( $i$ denotes the number of the reaction $\mathrm{R} i$ ) as proposed by Donlon and Novakovic. ${ }^{11}$ Concentrations of $\mathrm{CH}_{3} \mathrm{OH}, \mathrm{O}_{2}$, and $\mathrm{CO}$ are in excess, therefore, their concentrations may be considered constant and included in the rate constants $k_{i}$. (For these species, simple pool approximation is valid here.) The species $\mathrm{H}_{2} \mathrm{O}$ and PEGP are the products of reactions and consequently, they do not appear in the reaction rates. Here $c_{j}$ is the concentration of species $j$ denoted in the subscript with values 1-6 corresponding to the species $\mathrm{PdI}_{2}, \mathrm{Pd}, \mathrm{HI}, \mathrm{I}_{2}, \mathrm{IPdR}$, and PEGA, such that $c_{1}=c_{\mathrm{PdI}_{2}}, c_{2}=c_{\mathrm{Pd}}, c_{3}=c_{\mathrm{HI}}, c_{4}=c_{\mathrm{I}_{2}}, c_{5}=c_{\mathrm{IPdR}}$, and $c_{6}=c_{\mathrm{PEGA}}$.

\begin{tabular}{lll}
\hline $\mathrm{PEGA}+\mathrm{PdI}_{2}+2 \mathrm{HI}+2 \mathrm{CH}_{3} \mathrm{OH}$ & & \\
$\quad+2 \mathrm{CO} \rightarrow \mathrm{PEGP}+\mathrm{Pd}+4 \mathrm{HI}$ & $r_{1}=k_{1} c_{6} c_{1} c_{3}^{2}$ & $(\mathrm{R} 1)$ \\
$2 \mathrm{HI}+0.5 \mathrm{O}_{2} \rightarrow \mathrm{I}_{2}+\mathrm{H}_{2} \mathrm{O}$ & $r_{2}=k_{2} c_{3}^{2}$ & $(\mathrm{R} 2)$ \\
$\mathrm{Pd}+\mathrm{I}_{2} \rightarrow \mathrm{PdI}_{2}$ & $r_{3}=k_{3} c_{2} c_{4}$ & $(\mathrm{R} 3)$ \\
$\mathrm{Pd}+\mathrm{I}_{2}+\mathrm{PdI}_{2} \rightarrow 2 \mathrm{PdI}_{2}$ & $r_{4}=k_{4} c_{2} c_{4} c_{1}$ & $(\mathrm{R} 4)$ \\
$\mathrm{PdI}_{2}+\mathrm{CH}_{3} \mathrm{OH}+\mathrm{CO} \rightarrow \mathrm{IPdR}+\mathrm{HI}$ & $r_{5}=k_{5} c_{1}$ & $(\mathrm{R} 5)$ \\
$\mathrm{IPdR}+\mathrm{HI} \rightarrow \mathrm{PdI}_{2}+\mathrm{CH}_{3} \mathrm{OH}+\mathrm{CO}$ & $r_{6}=k_{6} c_{5} c_{3}$ \\
\hline
\end{tabular}

where $\mathrm{d} c / \mathrm{d} t$ is the time derivative of the concentration vector $\boldsymbol{c}, \boldsymbol{r}$ is the reaction rate vector, and $\mathbf{S}$ is the matrix of the stoichiometric coefficients obtained directly from the reaction network. ${ }^{17-20}$ In the case under consideration (Table I), the matrix $\mathbf{S}$ is

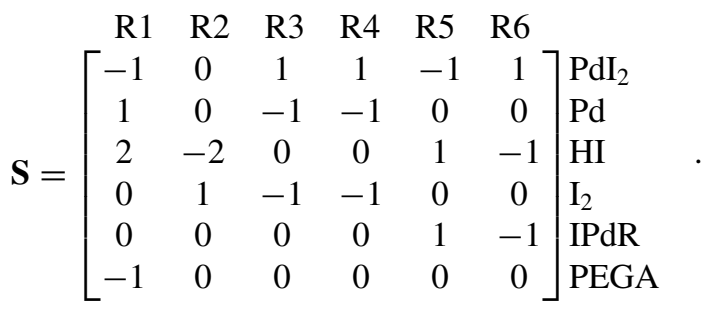

The species on the right hand side and reactions above the matrix are added for clarity and are not part of this matrix.

It is worth noting that in Eq. (1), the number of equations is defined by the reaction network, whereas the number of species under consideration is dependent on the SNA method used. We shall elaborate on this in Sec. III.

\section{SNA OF OSCILLATORY CARBONYLATION OF PEGA}

In SNA, it is very important to correctly select the kinetic equations to describe the dynamic state of the system. Hence, we need to revisit all the species operating in the case studied here and carefully consider their roles. Thus, in the reaction network presented in Table I, there are 11 chemical species: $\mathrm{CH}_{3} \mathrm{OH}, \mathrm{CO}, \mathrm{PEGP}, \mathrm{O}_{2}, \mathrm{H}_{2} \mathrm{O}$, PEGA, $\mathrm{PdI}_{2}, \mathrm{Pd}, \mathrm{HI}, \mathrm{I}_{2}$, and IPdR. Some of these species are present only as products, not involved in any further reactions, and therefore have no influence on reaction rates and dynamic states of the system. These species are $\mathrm{H}_{2} \mathrm{O}$ and PEGP. Furthermore, some species are present in nearly constant concentrations. Most often this is the case with reactants added in large surplus at the beginning of the reaction, like PEGA and $\mathrm{CH}_{3} \mathrm{OH}$, or reactants continuously fed, and in excess, to the reaction mixture, like $\mathrm{CO}$ and $\mathrm{O}_{2}$. Dynamic states depend on such reactants only in a weak, parametrical sense. As already mentioned, simple pool approximation is valid for these species. Only intermediate species are truly dynamical variables and state functions, so, 
only these species should be taken into account. The intermediate species in the reaction network under consideration here are $\mathrm{PdI}_{2}, \mathrm{Pd}, \mathrm{HI}, \mathrm{I}_{2}$, and IPdR. Thus, the corresponding matrix $\mathbf{S}$ has the form

$$
\mathbf{S}=\left[\begin{array}{cccccc}
-1 & 0 & 1 & 1 & -1 & 1 \\
1 & 0 & -1 & -1 & 0 & 0 \\
2 & -2 & 0 & 0 & 1 & -1 \\
0 & 1 & -1 & -1 & 0 & 0 \\
0 & 0 & 0 & 0 & 1 & -1
\end{array}\right] \begin{aligned}
& \mathrm{PdI}_{2} \\
& \mathrm{Pd} \\
& \mathrm{IPdR}
\end{aligned} .
$$

The time evolution and dynamic state of the reaction network (Table I) are now described in Eq. (5) by only the first five differential equations defined in Eq. (1).

$$
\left[\begin{array}{l}
\mathrm{d} c_{1} / \mathrm{d} t \\
\mathrm{~d} c_{2} / \mathrm{d} t \\
\mathrm{~d} c_{3} / \mathrm{d} t \\
\mathrm{~d} c_{4} / \mathrm{d} t \\
\mathrm{~d} c_{5} / \mathrm{d} t
\end{array}\right]=\left[\begin{array}{cccccc}
-1 & 0 & 1 & 1 & -1 & 1 \\
1 & 0 & -1 & -1 & 0 & 0 \\
2 & -2 & 0 & 0 & 1 & -1 \\
0 & 1 & -1 & -1 & 0 & 0 \\
0 & 0 & 0 & 0 & 1 & -1
\end{array}\right]\left[\begin{array}{l}
r_{1} \\
r_{2} \\
r_{3} \\
r_{4} \\
r_{5} \\
r_{6}
\end{array}\right]
$$

For stability analysis of the reaction network, the basic steady state must be defined. Therefore, the reaction rates in the steady state, $\boldsymbol{r}_{\mathrm{ss}}$, are necessary. They are solutions of the relation

$$
\mathrm{Sr}_{\mathrm{ss}}=0
$$

These rates at the steady state $\boldsymbol{r}_{\mathrm{ss}}$ can be expressed by means of the current rates $j_{\mathrm{i}}$, which are the contributions of the elementary reaction pathways with non-negative coefficients. ${ }^{1,21,22}$ The overall process can be represented as a linear combination of several elementary reaction pathways known as extreme currents $E_{i}$ and they all contribute to the steady-state values of reaction rates. The contributions of the extreme currents $E_{i}$, denoted as the current rates $j_{\mathrm{i}}$, are the components of the corresponding current rate vector $\boldsymbol{j}$, whereas the extreme currents $E_{\mathrm{i}}$ are the columns of the extreme current matrix $\mathbf{E}^{1,2,6,17,18,20-24}$ Thus,

$$
\boldsymbol{r}_{s s}=\mathbf{E} \mathbf{j},
$$

which is the basic equation in SNA. Now, we are ready to calculate extreme currents $E_{i}$ and discuss the stability of the basic steady state. However, although we selected only intermediate species to examine dynamic states of the considered reaction network, the matrix $\mathbf{S}$, given in Eq. (4), has a rank equal to 3 , indicating that two rate equations are linearly dependent on the other equations in the system. This phenomenon is due to two mass conservation constraints and neither of these species may be considered in high excess. Hence, pool approximation is not valid for these species.

Section III A (first method) will give the SNA procedure proposed for examination of systems where the concentrations of independent intermediate species are separated from dependent ones and conservation constraints are taken into account. In Sec. III B (second method), the standard procedure for examination of the instability condition will be applied to the full set of intermediate species.

\section{A. First method-SNA with independent intermediate species and conservation constraints}

Aiming to separate independent from dependent variables, two conservation constraints, given as two algebraic equations, appropriate for the reaction system studied are used. Two constraints, representing the conservation of palladium (Pd) [Eq. (8)] and iodine (I) [Eq. (9)] atoms, serve to express the concentrations of two dependant species as a function of the remaining independent ones.

$$
\begin{gathered}
c_{1}+c_{2}+c_{5}=\text { const }=c_{\text {tot }}(\mathrm{Pd}), \\
2 c_{1}+c_{3}+2 c_{4}+c_{5}=\text { const }=c_{\text {tot }}(\mathrm{I}) .
\end{gathered}
$$

For any given case, the conservation constraint can be expressed in the matrix form

$$
\gamma \boldsymbol{c}=\boldsymbol{c}_{\mathrm{tot}},
$$

where $\boldsymbol{\gamma}$ is the conservation matrix and $\boldsymbol{c}_{\text {tot }}$ is the constant vector with total concentrations of the conserved species. Moreover, the concentration of linearly dependent species can be separated from the independent ones in the form

$$
\gamma_{\mathrm{I}} \boldsymbol{c}_{\mathrm{I}}+\gamma_{\mathrm{D}} \boldsymbol{c}_{\mathrm{D}}=\boldsymbol{c}_{\mathrm{tot}},
$$

where subscripts I and D denote the independent and dependent species, respectively. Then, the dependent variables can be expressed as functions of the independent ones in the form

$$
\boldsymbol{c}_{\mathrm{D}}=\gamma_{\mathrm{D}}^{-1} \boldsymbol{c}_{\mathrm{tot}}-\gamma_{\mathrm{D}}^{-1} \gamma_{\mathrm{I}} \boldsymbol{c}_{\mathrm{I}} .
$$

In the case under consideration, we can choose, without losing generality, the two dependent species to be $\mathrm{I}_{2}$ and IPdR. Then, the conservation laws [Eqs. (8) and (9)] can be expressed in the following matrix form [Eq. (13)].

$$
\left[\begin{array}{lll}
1 & 1 & 0 \\
2 & 0 & 1
\end{array}\right]\left[\begin{array}{l}
c_{1} \\
c_{2} \\
c_{3}
\end{array}\right]+\left[\begin{array}{ll}
0 & 1 \\
2 & 1
\end{array}\right]\left[\begin{array}{l}
c_{4} \\
c_{5}
\end{array}\right]=\left[\begin{array}{c}
c_{\text {tot }}(\mathrm{Pd}) \\
c_{\text {tot }}(\mathrm{I})
\end{array}\right] .
$$

The dependent species can be expressed as given in Eq. (14).

$$
\begin{aligned}
{\left[\begin{array}{l}
c_{4} \\
c_{5}
\end{array}\right]=} & {\left[\begin{array}{cc}
-\frac{1}{2} & \frac{1}{2} \\
1 & 0
\end{array}\right]\left[\begin{array}{c}
c_{\text {tot }}(\mathrm{Pd}) \\
c_{\text {tot }}(\mathrm{I})
\end{array}\right] } \\
& -\left[\begin{array}{cc}
-\frac{1}{2} & \frac{1}{2} \\
1 & 0
\end{array}\right]\left[\begin{array}{lll}
1 & 1 & 0 \\
2 & 0 & 1
\end{array}\right]\left[\begin{array}{l}
c_{1} \\
c_{2} \\
c_{3}
\end{array}\right] .
\end{aligned}
$$

The problem to be solved now consists of independent equations only [Eq. (15)].

$$
\left[\begin{array}{l}
\mathrm{d} c_{1} / \mathrm{d} t \\
\mathrm{~d} c_{2} / \mathrm{d} t \\
\mathrm{~d} c_{3} / \mathrm{d} t
\end{array}\right]=\left[\begin{array}{cccccc}
-1 & 0 & 1 & 1 & -1 & 1 \\
1 & 0 & -1 & -1 & 0 & 0 \\
2 & -2 & 0 & 0 & 1 & -1
\end{array}\right]\left[\begin{array}{l}
r_{1} \\
r_{2} \\
r_{3} \\
r_{4} \\
r_{5} \\
r_{6}
\end{array}\right]
$$

Evidently, the matrix $\mathbf{S}_{\mathrm{I}}$ related to the independent species only is given by the equation:

$$
\mathbf{S}_{\mathrm{I}}=\left[\begin{array}{cccccc}
-1 & 0 & 1 & 1 & -1 & 1 \\
1 & 0 & -1 & -1 & 0 & 0 \\
2 & -2 & 0 & 0 & 1 & -1
\end{array}\right] \begin{aligned}
& \mathrm{PdI}_{2} \\
& \mathrm{Pd} \\
& \mathrm{HI}
\end{aligned} .
$$


The extreme current matrix $\mathbf{E}$ calculated by means of $\mathbf{S}_{\mathrm{I}}$ is

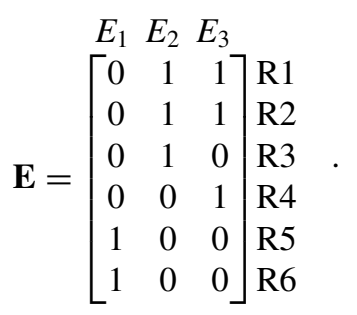

In this matrix, $E_{1}, E_{2}$, and $E_{3}$ are the extreme currents denoting elementary reaction pathways in the considered reaction network. By analyzing the extreme current matrix $\mathbf{E}$, the following net reactions were obtained

$$
E_{1}:(R 5)+(R 6) \Rightarrow 0 \rightarrow 0
$$

$$
\begin{aligned}
& E_{2}:(R 1)+(R 2)+(R 3) \Rightarrow \\
& \quad \mathrm{PEGA}+2 \mathrm{CH}_{3} \mathrm{OH}+2 \mathrm{CO}+0.5 \mathrm{O}_{2} \rightarrow \mathrm{PEGP}+\mathrm{H}_{2} \mathrm{O},
\end{aligned}
$$

$$
\begin{aligned}
& E_{3}:(R 1)+(R 2)+(R 4) \Rightarrow \\
& \quad \mathrm{PEGA}+2 \mathrm{CH}_{3} \mathrm{OH}+2 \mathrm{CO}+0.5 \mathrm{O}_{2} \rightarrow \mathrm{PEGP}+\mathrm{H}_{2} \mathrm{O} .
\end{aligned}
$$

From Eqs. (18) to (20), it can be noted that the first net reaction represents the equilibration between reactions (R5) and (R6) and that there are two possible reaction pathways besides this chemical equilibrium which allow the same transformation of the reactants into the products. More details on the calculation of all extreme currents can be found elsewhere. ${ }^{1,21,22,25,26}$

As we already mentioned, the extreme currents are crucial for finding the relationship between steady-state reaction rates and current rates. Thus, using the basic equation of the SNA [Eq. (7)], we can easily obtain steady-state reaction rates $\boldsymbol{r}_{\mathrm{ss}}$ as a function of current rates $j_{i}$, where $i=1,2,3$,

$$
\begin{aligned}
& r_{1, \mathrm{ss}}=j_{2}+j_{3}, \\
& r_{2, \mathrm{ss}}=j_{2}+j_{3}, \\
& r_{3, \mathrm{ss}}=j_{2}, \\
& r_{4, \mathrm{ss}}=j_{3}, \\
& r_{5, \mathrm{ss}}=j_{1}, \\
& r_{6, \mathrm{ss}}=j_{1} .
\end{aligned}
$$

Moreover, the significance of extreme currents is in their role in analysis of stability of the basic state. The stability of a steady state is usually examined through the response of the reaction system to some arbitrary small perturbation. The time evolution of small concentration perturbations $\Delta \boldsymbol{c}=\boldsymbol{c}-\boldsymbol{c}_{\mathrm{ss}}$ near a steady state $\boldsymbol{c}_{\mathrm{ss}}$ is given by Eq. (22).

$$
\mathrm{d} \Delta \boldsymbol{c} / \mathrm{d} t=\mathbf{M} \Delta \boldsymbol{c} .
$$

It was obtained by linearization of the general equation of motion around the steady state, and matrix $\mathbf{M}$ is the Jacobian of the reaction system given by Eq. (23).

$$
\mathbf{M}\left(\boldsymbol{r}_{\mathrm{ss}}\right)=\mathbf{S}\left(\operatorname{diag} \boldsymbol{r}_{\mathrm{ss}}\right) \mathbf{K}^{\mathrm{T}}\left(\operatorname{diag} \boldsymbol{c}_{s s}^{-1}\right)=\mathbf{S}[\operatorname{diag}(\mathrm{Ej})] \mathbf{K}^{\mathrm{T}} \operatorname{diag} \boldsymbol{h},
$$

where $\boldsymbol{h}$ stands for a vector of reciprocal steady-state concentrations of the intermediate species and $\operatorname{diag} \boldsymbol{h}$ is its diagonal matrix, while $\mathbf{K}$ is the matrix of the orders of reaction and $\mathbf{K}^{\mathrm{T}}$ is its transpose. If we assume mass action law for the reaction rates, the elements of matrix $\mathbf{K}$ are stoichiometric coefficients of a species standing on the left side of the particular reaction step (Table I).

The matrix M, written in the SNA, ${ }^{1}$ as a function of the SNA parameters $j_{i}$ and $h_{i}$ has particular advantages for the stability analysis since the parameters $j_{i}$ and $h_{i}$ are non-negative, which is an essential feature of the SNA. The steady-state stability is determined by the eigenvalues of $\mathbf{M}$, which are the roots $\lambda$ of the characteristic polynomial

$$
\begin{aligned}
\operatorname{Det}[\lambda \mathbf{I}-\mathbf{M}]= & \sum_{i=0}^{n} \alpha_{i} \lambda^{n-i}=\lambda^{\mathrm{n}}+\alpha_{1} \lambda^{\mathrm{n}-1}+\alpha_{2} \lambda^{\mathrm{n}-2} \\
& +\cdots+\alpha_{\mathrm{n}}=0,
\end{aligned}
$$

where $n$ is the number of independent intermediate species and coefficients $\alpha_{\mathrm{i}}$ are the sums of all diagonal minors of dimensions $i$ of matrix $\mathbf{M}$ multiplied by the product of the corresponding $h_{i}$ values. Each diagonal minor of the matrix $\mathbf{M}$, as the determinant of a square matrix having dimension $i$, can be formed by any combination of $i$ independent intermediate species. In one $\alpha_{i}$, the number of minors is equal to the number of different combinations of $i$ intermediates.

If the real parts of all eigenvalues are negative, a steady state is stable. If one or more eigenvalues have positive real parts, the steady state is unstable. The sign of the real part of the eigenvalues of the Jacobian matrix can be evaluated by using several criteria such as the Hurwitz determinants ${ }^{27,28}$ or $\alpha$-approximation. According to the $\alpha$-approximation, the eigenvalue with a positive real part occurs when some coefficient $\alpha$ of the characteristic polynomial is negative. ${ }^{1,27,28}$ The applicability of each approach depends on the model complexity.

Moreover, if we find negative minors in one coefficient $\alpha_{i}$ and we want to discuss its sign, then we ask if the sum of negative terms can be larger than the sum of positive terms in the same $\alpha_{i}{ }^{28-34}$

In our case, where we have dependent (D) and independent (I) variables, the Jacobian matrix M [Eq. (23)] can be divided into four parts, where the elements of the four parts $\mathbf{M}_{\mathrm{II}}, \mathbf{M}_{\mathrm{ID}}, \mathbf{M}_{\mathrm{DI}}$, and $\mathbf{M}_{\mathrm{DD}}$ of the Jacobian matrix are

$$
\begin{gathered}
\mathbf{M}_{\mathrm{II}}=\frac{\partial \frac{\mathrm{d} \Delta X_{\mathrm{I}}}{\mathrm{d} t} ;}{\partial \Delta X_{\mathrm{I}}} ; \quad \mathbf{M}_{\mathrm{ID}}=\frac{\partial \frac{\mathrm{d} \Delta X_{\mathrm{I}}}{\mathrm{d} t}}{\partial \Delta X_{\mathrm{D}}} ; \\
\mathbf{M}_{\mathrm{DI}}=\frac{\partial \frac{\mathrm{d} \Delta X_{\mathrm{D}}}{\mathrm{d}_{t}} ;}{\partial \Delta X_{\mathrm{I}}} ; \quad \mathbf{M}_{\mathrm{DD}}=\frac{\partial \frac{\mathrm{d} \Delta X_{\mathrm{D}}}{\mathrm{d} t}}{\partial \Delta X_{\mathrm{D}}} .
\end{gathered}
$$

Now, Eq. (22) can be written in the form

$$
\frac{\mathrm{d}}{\mathrm{d} t}\left[\begin{array}{c}
\Delta \boldsymbol{c}_{\mathrm{I}} \\
\Delta \boldsymbol{c}_{\mathrm{D}}
\end{array}\right]=\left[\begin{array}{ll}
\mathbf{M}_{\mathrm{II}} & \mathbf{M}_{\mathrm{ID}} \\
\mathbf{M}_{\mathrm{DI}} & \mathbf{M}_{\mathrm{DD}}
\end{array}\right]\left[\begin{array}{c}
\Delta \boldsymbol{c}_{\mathrm{I}} \\
\Delta \boldsymbol{c}_{\mathrm{D}}
\end{array}\right]
$$

and the dynamics of the independent species concentrations can be expressed from (26) by the following equation:

$$
\frac{\mathrm{d} \Delta \boldsymbol{c}_{\mathrm{I}}}{\mathrm{d} t}=\mathbf{M}_{\mathrm{II}} \Delta \boldsymbol{c}_{\mathrm{I}}+\mathbf{M}_{\mathrm{ID}} \Delta \boldsymbol{c}_{\mathrm{D}}
$$

Taking into account that $\Delta \boldsymbol{c}=\boldsymbol{c}-\boldsymbol{c}_{\mathrm{ss}}$ and Eq. (12), we can find

$$
\Delta \boldsymbol{c}_{\mathrm{D}}=\gamma_{\mathrm{D}}^{-1} \Delta \boldsymbol{c}_{\mathrm{tot}}-\gamma_{\mathrm{D}}^{-1} \gamma_{\mathrm{I}} \Delta \boldsymbol{c}_{\mathrm{I}}=-\gamma_{\mathrm{D}}^{-1} \gamma_{\mathrm{I}} \Delta \boldsymbol{c}_{\mathrm{I}},
$$


since $\Delta \boldsymbol{c}_{\mathrm{tot}}=0$. Substituting (28) into (27), one obtains

$$
\frac{\mathrm{d} \Delta \boldsymbol{c}_{\mathrm{I}}}{\mathrm{d} t}=\mathbf{M}^{*} \Delta \boldsymbol{c}_{\mathrm{I}}
$$

where the Jacobian matrix $\mathbf{M}^{*}$ is given in Eq. (30).

$$
\mathbf{M}^{*}=\mathbf{M}_{\mathrm{II}}-\mathbf{M}_{\mathrm{ID}} \gamma_{\mathrm{D}}^{-1} \gamma_{\mathrm{I}} \text {. }
$$

Considering Eqs. (23) and (26), the matrices in Eq. (30) may be identified as

$$
\mathbf{M}_{\mathrm{II}}(\boldsymbol{j}, \boldsymbol{h})=\mathbf{S}_{\mathrm{I}}(\operatorname{diag} \mathbf{E} \boldsymbol{j})\left[\mathbf{K}_{\mathrm{I}}^{\mathrm{T}}\left(\operatorname{diag} \boldsymbol{h}_{\mathrm{I}}\right)\right]
$$

and

$$
\mathbf{M}_{\mathrm{ID}}(\boldsymbol{j}, \boldsymbol{h})=\mathbf{S}_{\mathrm{I}}(\operatorname{diag} \mathbf{E} \boldsymbol{j})\left[\mathbf{K}_{\mathrm{D}}^{\mathrm{T}}\left(\operatorname{diag} \boldsymbol{h}_{\mathrm{D}}\right)\right]
$$

so that we finally obtain ${ }^{1}$

$$
\mathbf{M}^{*}(\boldsymbol{j}, \boldsymbol{h})=\mathbf{S}_{\mathrm{I}}(\operatorname{diag} \mathbf{E} \boldsymbol{j})\left[\mathbf{K}_{\mathrm{I}}^{\mathrm{T}}\left(\operatorname{diag} \boldsymbol{h}_{\mathrm{I}}\right)-\mathbf{K}_{\mathrm{D}}^{\mathrm{T}}\left(\operatorname{diag} \boldsymbol{h}_{\mathrm{D}}\right) \gamma_{\mathrm{D}}^{-1} \gamma_{\mathrm{I}}\right] .
$$

This is the form of the Jacobian matrix which should be analysed in the case when some intermediate species are dependent on others, instead of the Jacobian matrix $\mathbf{M}$ given in Eq. (23).

For the reaction network analysed here (Table I), the matrix $\mathbf{S}_{\mathrm{I}}$ is given in Eq. (16), whereas the matrix of reaction orders $\mathbf{K}$ for the independent and dependent species $\mathbf{K}_{\mathrm{I}}$ and $\mathbf{K}_{\mathrm{D}}$, respectively, are given in the following equations:

$$
\begin{aligned}
& \mathbf{K}_{\mathrm{I}}=\left[\begin{array}{llllll}
1 & 0 & 0 & 1 & 1 & 0 \\
0 & 0 & 1 & 1 & 0 & 0 \\
2 & 2 & 0 & 0 & 0 & 1
\end{array}\right] \begin{array}{l}
\mathrm{PdI}_{2} \\
\mathrm{Pd} \\
\mathrm{HI}
\end{array}, \\
& \mathbf{K}_{\mathrm{D}}=\left[\begin{array}{llllll}
0 & 0 & 1 & 1 & 0 & 0 \\
0 & 0 & 0 & 0 & 0 & 1
\end{array}\right] \mathrm{I}_{2} \mathrm{IPdR}
\end{aligned} .
$$

Now we can use Eq. (33) to obtain the complete Jacobian matrix [Eq. (36)]

$$
\mathbf{M}^{*}(\boldsymbol{j}, \boldsymbol{h})=\left[\begin{array}{lll}
m_{1,1} & m_{1,2} & m_{1,3} \\
m_{2,1} & m_{2,2} & m_{2,3} \\
m_{3,1} & m_{3,2} & m_{3,3}
\end{array}\right]
$$

with coefficients $m_{\mathrm{p}, \mathrm{q}}$ equal to

$$
\begin{aligned}
& m_{1,1}=j_{3}\left(h_{1}-\frac{h_{4}}{2}\right)-\frac{\left(h_{4} j_{2}\right)}{2}-h_{5} j_{1}-h_{1} j_{1}-h_{1}\left(j_{2}+j_{3}\right), \\
& m_{1,2}=j_{2}\left(h_{2}+\frac{h_{4}}{2}\right)-h_{5} j_{1}+j_{3}\left(h_{2}+\frac{h_{4}}{2}\right), \\
& m_{1,3}=h_{3} j_{1}-\frac{h_{4} j_{2}}{2}-\frac{h_{4} j_{3}}{2}-2 h_{3}\left(j_{2}+j_{3}\right), \\
& m_{2,1}=\frac{h_{4} j_{2}}{2}-j_{3}\left(h_{1}-\frac{h_{4}}{2}\right)+h_{1}\left(j_{2}+j_{3}\right),
\end{aligned}
$$

$$
\begin{aligned}
& m_{2,2}=-j_{2}\left(h_{2}+\frac{h_{4}}{2}\right)-j_{3}\left(h_{2}+\frac{h_{4}}{2}\right), \\
& m_{2,3}=\frac{h_{4} j_{2}}{2}+\frac{h_{4} j_{3}}{2}+2 h_{3}\left(j_{2}+j_{3}\right) \\
& m_{3,1}=h_{1} j_{1}+h_{5} j_{1}+h_{1}\left(2 j_{2}+2 j_{3}\right) \\
& m_{3,2}=h_{5} j_{1} \\
& m_{3,3}=-h_{3} j_{1} .
\end{aligned}
$$

The obtained alpha coefficients are

$$
\begin{aligned}
\alpha_{1}=h_{1} j_{1} & +h_{1} j_{2}+h_{2} j_{2}+h_{3} j_{1}+h_{2} j_{3}+h_{4} j_{2}+h_{5} j_{1}+h_{4 j_{3}}, \\
\alpha_{2}= & 4 h_{1} h_{3} j_{2}{ }^{2}+4 h_{1} h_{3} j_{3}^{2}+h_{1} h_{4} j_{2}^{2}+h_{1} h_{4} j_{3}^{2} \\
& +h_{1} h_{2} j_{1} j_{2}+h_{1} h_{2} j_{1} j_{3}+h_{1} h_{3} j_{1} j_{2}+h_{1} h_{4} j_{1} j_{2} \\
& +h_{2} h_{3} j_{1} j_{2}+8 h_{1} h_{3} j_{2} j_{3}+h_{1} h_{4} j_{1} j_{3}+h_{1} h_{5} j_{1} j_{2} \\
& +h_{2} h_{3} j_{1} j_{3}+2 h_{1} h_{4} j_{2} j_{3}+h_{2} h_{5} j_{1} j_{2}+h_{3} h_{4} j_{1} j_{2} \\
& +h_{2} h_{5} j_{1} j_{3}+h_{3} h_{4} j_{1} j_{3}+h_{4} h_{5} j_{1} j_{2}+h_{4} h_{5} j_{1} j_{3}
\end{aligned}
$$

Since there are negative terms in the largest diagonal minor of the Jacobian matrix $\mathbf{M}^{*}$, having dimension $3 \times 3$, the characteristic polynomial $\alpha_{3}(\boldsymbol{j}, \boldsymbol{h})$, can also be negative, allowing the generation of instability. As $\alpha_{1}(\boldsymbol{j}, \boldsymbol{h})$ and $\alpha_{2}(\boldsymbol{j}, \boldsymbol{h})$ are both positive, we are dealing with a saddle point. At this level of approximation, the system becomes unstable when $\alpha_{3}$ becomes negative, and this situation occurs when

$$
2 h_{2} h_{3}+h_{3} h_{4}>4 h_{3} h_{5}+h_{4} h_{5} .
$$

We can evaluate concentrations at the steady states and their corresponding reciprocals $h_{\mathrm{i}}$ to find which states are stable and which are not (Appendix).

Now, we shall analyse the instability criteria by the other procedure mentioned in the Introduction and given in Sec. III B.

\section{B. Second method-SNA with the full set of intermediate species}

In this method, we will perform the calculation using all five dynamical variables, namely, the concentrations of the intermediate species $\mathrm{PdI}_{2}, \mathrm{Pd}, \mathrm{HI}, \mathrm{I}_{2}$, and IPdR. In other words, we will not split the independent and dependent variables. Thus, the extreme currents $E_{i}$ will be calculated using the $\mathbf{S}$ matrix given in Eq. (4). The result for the extreme current matrix, E, is equal to that obtained by the first method [see Eq. (17)]. It means that Eqs. (18)-(21) are also the same.

Aiming to discuss stability, we shall calculate matrix $\mathbf{M}$ from Eq. (23) using the following matrix of reaction orders $\mathbf{K}$

$$
\mathbf{K}=\left[\begin{array}{llllll}
1 & 0 & 0 & 1 & 1 & 0 \\
0 & 0 & 1 & 1 & 0 & 0 \\
2 & 2 & 0 & 0 & 0 & 1 \\
0 & 0 & 1 & 1 & 0 & 0 \\
0 & 0 & 0 & 0 & 0 & 1
\end{array}\right] \begin{aligned}
& \mathrm{PdI}_{2} \\
& \mathrm{Pd} \\
& \mathrm{HI} \\
& \mathrm{I}_{2} \\
& \mathrm{IPdR}
\end{aligned}
$$

The obtained matrix $\mathbf{M}$ is 


$$
\mathbf{M}(j)=\left[\begin{array}{cc}
-h_{1}\left(j_{1}+j_{2}\right) & h_{2}\left(j_{2}+j_{3}\right) \\
h_{1} j_{2} & -h_{2}\left(j_{2}+j_{3}\right) \\
h_{1}\left(j_{1}+2 j_{2}+2 j_{3}\right) & 0 \\
-h_{1} j_{3} & -h_{2}\left(j_{2}+j_{3}\right) \\
h_{1} j_{1} & 0
\end{array}\right.
$$

The corresponding alpha coefficients are given in the next five equations:

$$
\begin{aligned}
\alpha_{1}= & h_{1} j_{1}+h_{1} j_{2}+h_{2} j_{2}+h_{3} j_{1}+h_{2} j_{3}+h_{4} j_{2}+h_{5} j_{1}+h_{4} j_{3}, \\
\alpha_{2}= & 4 h_{1} h_{3} j_{2}^{2}+4 h_{1} h_{3} j_{3}^{2}+h_{1} h_{4} j_{2}^{2}+h_{1} h_{4} j_{3}^{2}+h_{1} h_{2} j_{1} j_{2} \\
& +h_{1} h_{2} j_{1} j_{3}+h_{1} h_{3} j_{1} j_{2}+h_{1} h_{4} j_{1} j_{2}+h_{2} h_{3} j_{1} j_{2}+8 h_{1} h_{3} j_{2} j_{3} \\
& +h_{1} h_{4} j_{1} j_{3}+h_{1} h_{5} j_{1} j_{2}+h_{2} h_{3} j_{1} j_{3}+2 h_{1} h_{4} j_{2} j_{3}+h_{2} h_{5} j_{1} j_{2} \\
& +h_{3} h_{4} j_{1} j_{2}+h_{2} h_{5} j_{1} j_{3}+h_{3} h_{4} j_{1} j_{3}+h_{4} h_{5} j_{1} j_{2}+h_{4} h_{5} j_{1} j_{3},
\end{aligned}
$$

$$
\begin{aligned}
& \alpha_{4}=0, \\
& \alpha_{5}=0 .
\end{aligned}
$$$$
\alpha_{3}=-h_{1} j_{1}\left(j_{2}+j_{3}\right)^{2}\left(2 h_{2} h_{3}+h_{3} h_{4}-4 h_{3} h_{5}-h_{4} h_{5}\right),
$$

The coefficients $\alpha_{4}$ and $\alpha_{5}$ are equal to zero because we are dealing with a model where concentrations of only three species are independent ones. The coefficients $\alpha_{1}, \alpha_{2}$, and $\alpha_{3}$ are equal to the ones obtained by the first procedure. Thus, we have obtained the same results by both procedures explained above.

\section{DISCUSSION}

The fact that we obtained the same results by both procedures applied to the same model is logical if the methods are correctly postulated and correctly used. This statement can be confirmed mathematically. It is well known that the set of differential equations which belong to the model with conservation relations can be rewritten in the new one where these relations are included to replace dependant variables. ${ }^{23,35}$ Thus, each conservation law reduces the degree of the characteristic polynomial by one. In the case of the model presented here, there are two conservation laws, so the $5 \times 5$ Jacobian leads to an effective cubic order $(5-2=3)$ characteristic polynomial. Finally, in the case under consideration, the new set of differential equations obtained after incorporation of two conservation relations into the initial set of five differential equations (5) is

$$
\begin{aligned}
\frac{\mathrm{d} c_{1}}{\mathrm{~d} t}= & -k_{1} c_{6} c_{1} c_{3}^{2}+\frac{1}{2}\left(k_{3} c_{2}+k_{4} c_{2} c_{1}\right) \\
& \left(c_{\mathrm{tot}}(\mathrm{I})-c_{\mathrm{tot}}(\mathrm{Pd})-c_{1}+c_{2}-c_{3}\right)-k_{5} c_{1} \\
& +k_{6} c_{3}\left(c_{\mathrm{tot}}(\mathrm{Pd})-c_{1}-c_{2}\right),
\end{aligned}
$$

$$
\left.\begin{array}{ccc}
-h_{3}\left(2 j_{2}-j_{1}+2 j_{3}\right) & h_{4}\left(j_{2}+j_{3}\right) & h_{5} j_{1} \\
h_{3}\left(2 j_{2}+2 j_{3}\right) & -h_{4}\left(j_{2}+j_{3}\right) & 0 \\
-h_{3} j_{1} & 0 & -h_{5} j_{1} \\
h_{3}\left(2 j_{2}+2 j_{3}\right) & -h_{4}\left(j_{2}+j_{3}\right) & 0 \\
-h_{3} j_{1} & 0 & -h_{5} j_{1}
\end{array}\right] .
$$

$$
\begin{aligned}
\frac{\mathrm{d} c_{2}}{\mathrm{~d} t}= & k_{1} c_{6} c_{1} c_{3}^{2}-\frac{1}{2}\left(k_{3} c_{2}+k_{4} c_{2} c_{1}\right) \\
& \left(c_{\mathrm{tot}}(\mathrm{I})-c_{\mathrm{tot}}(\mathrm{Pd})-c_{1}+c_{2}-c_{3}\right), \\
\frac{\mathrm{d} c_{3}}{\mathrm{~d} t}= & 2 k_{1} c_{6} c_{1} c_{3}^{2}-2 k_{2} c_{3}^{2}+k_{5} c_{1}-k_{6} c_{3}\left(c_{\mathrm{tot}}(\mathrm{Pd})-c_{1}-c_{2}\right) .
\end{aligned}
$$

Thus, we obtained the system of three differential equations without additional relations for conservation constraints. In the new set of differential equations, there are 15 distinct monomial terms. Moreover, these terms are not completely independent, since they involve the same rate constants in a repeating manner. Therefore, the number of combinations of these monomial terms which could possibly result in steady states is only apparently higher than in the original system. An additional problem, in this case, could arise from false negative terms. Namely, when the concentrations of dependent variables are replaced by independent ones using equations similar to Eq. (14), several negative terms occur in the resulting expression of the form $c_{\mathrm{i}}=c_{t o t}-\sum_{j \neq i} s_{\mathrm{j}} c_{\mathrm{j}}$, but their algebraic sum is always positive since negative concentrations would have no physical meaning.

The generality of the statement that both mathematical procedures applied to the same model give equal result can be illustrated briefly by three additional examples. In the first two discussed by Aguda and Clarke ${ }^{36}$ and by Domijan and Kirkilionis ${ }^{23}$ there is a saddle node, whereas in the third one, ${ }^{23}$ there is an Andronov-Hopf bifurcation.

First example-This example is related to the reversible classical substrate-inhibition enzyme mechanism elaborated by Aguda and Clarke. ${ }^{36}$ The dynamic state of the overall process is described by the following set of differential equations

$$
\begin{aligned}
{\left[\begin{array}{c}
\frac{\mathrm{d} x_{1}}{\mathrm{~d} t} \\
\frac{\mathrm{d} x_{2}}{\mathrm{~d} t} \\
\frac{\mathrm{d} x_{3}}{\mathrm{~d} t} \\
\mathrm{~d} x_{4} \\
\hline \mathrm{d} t
\end{array}\right]=} & {\left[\begin{array}{cccccccc}
0 & 0 & -1 & 1 & 0 & 0 & 1 & -1 \\
1 & -1 & -1 & 0 & -1 & 1 & 1 & 0 \\
0 & 0 & 1 & -1 & -1 & 1 & -1 & 1 \\
0 & 0 & 0 & 0 & 1 & -1 & 0 & 0
\end{array}\right] } \\
& \times\left[\begin{array}{c}
k_{1} \\
k_{2} x_{2} \\
k_{3} x_{1} x_{2} \\
k_{4} x_{3} \\
k_{5} x_{2} x_{3} \\
k_{6} x_{4} \\
k_{7} x_{3} \\
k_{8} x_{1}
\end{array}\right]=\mathbf{S}\left[\begin{array}{c}
r_{1} \\
r_{2} \\
r_{3} \\
r_{4} \\
r_{5} \\
r_{6} \\
r_{7} \\
r_{8}
\end{array}\right],
\end{aligned}
$$


where variables $x_{1}, \ldots, x_{4}$ are the concentrations of the free enzyme Z, substrate $\mathrm{U}$, and enzyme-substrate complexes ZU and $\mathrm{UZU}$, respectively. $\mathbf{S}$ is the matrix of the stoichiometric coefficients. Parameters $k_{\mathrm{ij}}$ are the rate constants of related reactions. In the model under consideration, there is one conservation relation, which is

$$
x_{1}+x_{3}+x_{4}=\text { const }=c_{t o t}(Z)
$$

The model is analysed by both mathematical procedures. In both cases, the extreme current matrix, E, and obtained alpha coefficients are equal. They are

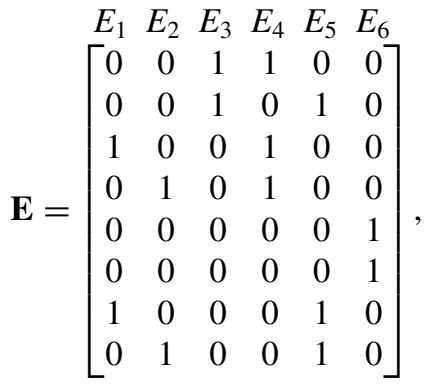

$$
\begin{aligned}
& \alpha_{1}=h_{4} j_{6}+h_{2}\left(j_{1}+j_{3}+j_{4}+j_{5}+j_{6}\right) \\
& +h_{3}\left(j_{1}+j_{2}+j_{4}+j_{5}+j_{6}\right)+h_{1}\left(j_{1}+j_{2}+j_{4}+j_{5}\right) \text {, } \\
& a_{2}=h_{2} h_{3}\left(j_{1} j_{2}+j_{1} j_{3}+j_{1} j_{4}+j_{2} j_{3}+j_{1} j_{5}+j_{2} j_{4}\right. \\
& +4 j_{1} j_{6}+j_{2} j_{5}+j_{3} j_{4}+j_{2} j_{6}+j_{3} j_{5}+j_{3} j_{6} \\
& \left.+j_{4} j_{5}+3 j_{4} j_{6}+3 j_{5} j_{6}+j_{4}^{2}+j_{5}^{2}\right)+h_{1} h_{2}\left(j_{1} j_{2}+j_{1} j_{3}\right. \\
& +j_{2} j_{3}+2 j_{1} j_{5}+j_{2} j_{4}+j_{1} j_{6}+j_{2} j_{5}+j_{3} j_{4}+j_{2} j_{6}+j_{3} j_{5} \\
& \left.+2 j_{4} j_{5}+j_{4} j_{6}+j_{5} j_{6}+j_{5}^{2}\right)+h_{1} h_{3} j_{6}\left(j_{1}+j_{2}+j_{4}+j_{5}\right) \\
& +h_{1} h_{4} j_{6}\left(j_{1}+j_{2}+j_{4}+j_{5}\right)+h_{2} h_{4} j_{6}\left(j_{1}+j_{3}+j_{4}+j_{5}\right) \\
& +h_{3} h_{4} j_{6}\left(j_{1}+j_{2}+j_{4}+j_{5}\right) \text {, } \\
& \alpha_{3}=h_{1} h_{2} h_{3} j_{6}\left(j_{1} j_{2}+j_{1} j_{3}-j_{1} j_{4}+j_{2} j_{3}+3 j_{1} j_{5}+2 j_{2} j_{5}+j_{3} j_{4}\right. \\
& \left.+j_{3} j_{5}+2 j_{4} j_{5}-j_{4}^{2}+2 j_{5}^{2}\right)+h_{2} h_{3} h_{4} j_{6}\left(j_{1} j_{2}+j_{1} j_{3}+j_{1} j_{4}\right. \\
& \left.+j_{2} j_{3}+j_{1} j_{5}+j_{2} j_{4}+j_{2} j_{5}+j_{3} j_{4}+j_{3} j_{5}+j_{4} j_{5}+j_{4}^{2}+j_{5}^{2}\right) \\
& +h_{1} h_{2} h_{4} j_{6}\left(j_{1} j_{2}+j_{1} j_{3}+j_{2} j_{3}+2 j_{1} j_{5}+j_{2} j_{4}+j_{2} j_{5}\right. \\
& +j_{3} j_{4}+j_{3} j_{5}+2 j_{4} j_{5}+j_{5}^{2} \text {. }
\end{aligned}
$$

Since there are negative terms in the largest diagonal minor of the Jacobian matrix $\mathbf{M}^{*}$, having dimension $3 \times 3$, the characteristic polynomial $\alpha_{3}(\boldsymbol{j}, \boldsymbol{h})$, can also be negative, allowing the generation of instability. As $\alpha_{1}(\boldsymbol{j}, \boldsymbol{h})$ and $\alpha_{2}(\boldsymbol{j}, \boldsymbol{h})$ are both positive, we are dealing with a saddle point.

Second example-This model related to a complex cell cycle $^{37}$ is elaborated from the mathematical point of view by Domijan and Kirkilionis. ${ }^{23}$ The dynamic state of the overall process is described by the following set of differential equations

$$
\begin{aligned}
{\left[\begin{array}{c}
\frac{\mathrm{d} x_{1}}{\mathrm{~d} t} \\
\frac{\mathrm{d} x_{2}}{\mathrm{~d} t} \\
\frac{\mathrm{d} x_{3}}{\mathrm{~d} t} \\
\frac{\mathrm{d} x_{4}}{\mathrm{~d} t} \\
\frac{\mathrm{d} x_{5}}{\mathrm{~d} t} \\
\frac{\mathrm{d} x_{6}}{\mathrm{~d} t}
\end{array}\right]=} & {\left[\begin{array}{cccccc}
0 & 0 & 1 & -1 & 0 & 0 \\
-1 & 1 & 0 & 0 & 0 & 0 \\
1 & -1 & 0 & 0 & 0 & 0 \\
0 & 0 & 0 & 0 & 1 & -1 \\
0 & 0 & -1 & 1 & 0 & 0 \\
0 & 0 & 0 & 0 & -1 & 1
\end{array}\right] } \\
\times & {\left[\begin{array}{c}
k_{1} x_{1} x_{2} \\
k_{2} x_{3} x_{4} \\
k_{3} x_{3} x_{5} \\
k_{4} x_{1} \\
k_{5} x_{6} \\
k_{6} x_{3} x_{4}
\end{array}\right]=\mathbf{S}\left[\begin{array}{c}
r_{1} \\
r_{2} \\
r_{3} \\
r_{4} \\
r_{5} \\
r_{6}
\end{array}\right], }
\end{aligned}
$$

where variables $x_{1}, \ldots, x_{4}$ are the concentrations of the main species in the system and parameters $k_{\mathrm{ij}}$ are the rate constants of related reactions. $\mathbf{S}$ is the matrix of the stoichiometric coefficients.

In the model under consideration, there are three conservation relations, which are

$$
\begin{aligned}
& x_{1}+x_{5}=a_{1}, \\
& x_{2}+x_{3}=a_{2}, \\
& x_{4}+x_{6}=a_{3} .
\end{aligned}
$$

The model is analysed by both mathematical procedures. In both cases, the extreme current matrix, E, and alpha coefficients are equal. They are

$$
\begin{gathered}
E_{1} E_{2} E_{3} \\
\mathbf{E}=\left[\begin{array}{lll}
0 & 1 & 0 \\
0 & 1 & 0 \\
1 & 0 & 0 \\
1 & 0 & 0 \\
0 & 0 & 1 \\
0 & 0 & 1
\end{array}\right] \\
\alpha_{1}=j_{1}\left(h_{1}+h_{5}\right)+j_{2}\left(h_{2}+h_{3}\right)+j_{3}\left(h_{4}+h_{6}\right) \\
=j_{1} j_{2}\left(h_{1} h_{2}+h_{2} h_{5}+h_{3} h_{5}\right)+j_{1} j_{3}\left(h_{1} h_{4}+h_{1} h_{6}+h_{4} h_{5}\right. \\
\left.+h_{5} h_{6}\right)+j_{2} j_{3}\left(h_{2} h_{4}+h_{2} h_{6}+h_{3} h_{6}\right) \\
\alpha_{3}=j_{1} j_{2} j_{3}\left(h_{1} h_{2} h_{4}-h_{1} h_{3} h_{4}+h_{1} h_{2} h_{6}\right. \\
\left.+h_{2} h_{4} h_{5}+h_{2} h_{5} h_{6}+h_{3} h_{5} h_{6}\right)
\end{gathered}
$$

Here, as in the previous example, the characteristic polynomial $\alpha_{3}(\boldsymbol{j}, \boldsymbol{h})$, can also be negative, allowing the generation of instability. As $\alpha_{1}(\boldsymbol{j}, \boldsymbol{h})$ and $\alpha_{2}(\boldsymbol{j}, \boldsymbol{h})$ are both positive, we are dealing with a saddle point. The conclusion is in accordance with the result obtained in Domijan and Kirkilionis. ${ }^{23}$

Third example - This model relates to intracellular calcium oscillations describing the enzymatic transfer of calcium ions $\mathrm{Ca}^{2+}$ across the cell membrane. ${ }^{35}$ The dynamic state 
of the overall process is described by the following set of differential equations

$$
\begin{aligned}
{\left[\begin{array}{c}
\frac{\mathrm{d} x_{1}}{\mathrm{~d} t} \\
\mathrm{~d} x_{2} \\
\mathrm{~d} t \\
\frac{\mathrm{d} x_{3}}{\mathrm{~d} t} \\
\mathrm{~d} x_{4} \\
\mathrm{~d} t
\end{array}\right]=} & {\left[\begin{array}{cccccc}
-1 & 1 & 1 & 1 & -1 & 0 \\
0 & 0 & -1 & 0 & 0 & 1 \\
0 & 0 & 0 & 1 & -1 & 1 \\
0 & 0 & 0 & -1 & 1 & -1
\end{array}\right] } \\
& \times\left[\begin{array}{c}
k_{12} x_{1} \\
k_{21} \\
k_{43} x_{1} x_{2} \\
k_{56} x_{4} \\
k_{65} x_{1} x_{3} \\
k_{76} x_{4}
\end{array}\right]=\mathbf{S}\left[\begin{array}{l}
r_{1} \\
r_{2} \\
r_{3} \\
r_{4} \\
r_{5} \\
r_{6}
\end{array}\right],
\end{aligned}
$$

where variables $x_{1}, \ldots, x_{4}$ are the concentrations of the main species in the system and parameters $k_{\mathrm{ij}}$ are the rate constants of related reactions. $\mathbf{S}$ is the matrix of the stoichiometric coefficients. The explanation of the process and physical meaning of variables and parameters are given in Domijan and Kirkilionis. ${ }^{23}$

In the considered model, there is one conservation relation, which is

$$
x_{3}+x_{4}=\text { const }=a .
$$

The model is analysed by both mathematical procedures. In both cases, the extreme current matrix, $\mathbf{E}$, and alpha coefficients are equal. They are

$$
\begin{aligned}
& \mathbf{E}=\left[\begin{array}{ccc}
E_{1} & E_{2} & E_{3} \\
1 & 0 & 0 \\
1 & 0 & 0 \\
0 & 0 & 1 \\
0 & 1 & 0 \\
0 & 1 & 1 \\
0 & 0 & 1
\end{array}\right], \\
& \alpha_{1}=h_{1}\left(j_{1}+j_{2}\right)+h_{2} j_{3}+\left(h_{3}+h_{4}\right)\left(j_{2}+j_{3}\right) \text {, } \\
& \alpha_{2}=h_{1} h_{2} j_{3}\left(j_{1}+j_{2}+j_{3}\right)+\left(h_{1} h_{4} j_{1}+\left(h_{2} h_{3}+h_{2} h_{4}\right) j_{3}\right. \\
& \left.+h_{1} h_{3}\left(j_{1}-j_{3}\right)\right)\left(j_{2}+j_{3}\right) \text {, } \\
& \alpha_{3}=h_{1} h_{2} j_{1} j_{3}\left(h_{3}+h_{4}\right)\left(j_{2}+j_{3}\right) \text {. }
\end{aligned}
$$

Since there are negative terms in $\alpha_{2}(\boldsymbol{j}, \boldsymbol{h})$, whereas $\alpha_{1}(\boldsymbol{j}, \boldsymbol{h})$ and $\alpha_{3}(\boldsymbol{j}, \boldsymbol{h})$ are always positive, we are dealing with the Andronov-Hopf bifurcation in accordance with the result obtained in Domijan and Kirkilionis. ${ }^{23}$

Obtaining the same result for both mathematical procedures may be justified by the following reasoning. In the second (simpler) method, eigenvalues $\lambda$-roots of the characteristic polynomial—are determined for the linearized operator, the Jacobian matrix M [Eq. (23)], in its full size:

$$
\mathbf{M x}=\lambda \mathbf{x},
$$

where $\mathbf{x}$ is a matrix with rows composed of eigenvectors corresponding to eigenvalues $\lambda$. In developed form, Eq. (69) reads

$$
\left[\begin{array}{ll}
\mathbf{M}_{\mathrm{II}} & \mathbf{M}_{\mathrm{ID}} \\
\mathbf{M}_{\mathrm{DI}} & \mathbf{M}_{\mathrm{DD}}
\end{array}\right]\left[\begin{array}{c}
x_{\mathrm{I}} \\
x_{\mathrm{D}}
\end{array}\right]=\left[\begin{array}{cc}
\lambda_{\mathrm{I}} & 0 \\
0 & \lambda_{\mathrm{D}}
\end{array}\right]\left[\begin{array}{l}
x_{\mathrm{I}} \\
x_{\mathrm{D}}
\end{array}\right],
$$

where again, $x_{\mathrm{I}}$ and $x_{\mathrm{D}}$ are matrices with rows composed of eigenvectors corresponding to eigenvalues $\lambda_{\mathrm{I}}$ and $\lambda_{\mathrm{D}}$. Due to linear dependence induced by conservation laws, all corresponding eigenvalues $\lambda_{\mathrm{D}}$ must be equal to zero. The resulting form of the problem is then condensed to

$$
\left[\begin{array}{ll}
\mathbf{M}_{\mathrm{II}} & \mathbf{M}_{\mathrm{ID}} \\
\mathbf{M}_{\mathrm{DI}} & \mathbf{M}_{\mathrm{DD}}
\end{array}\right]\left[\begin{array}{c}
x_{\mathrm{I}} \\
x_{\mathrm{D}}
\end{array}\right]=\left[\begin{array}{cc}
\lambda_{\mathrm{I}} & 0 \\
0 & 0
\end{array}\right]\left[\begin{array}{c}
x_{\mathrm{I}} \\
x_{\mathrm{D}}
\end{array}\right] .
$$

Equation (71) may be divided into two parts

$$
\begin{gathered}
{\left[\begin{array}{ll}
\mathbf{M}_{\mathrm{II}} & \mathbf{M}_{\mathrm{ID}}
\end{array}\right]\left[\begin{array}{c}
x_{\mathrm{I}} \\
x_{\mathrm{D}}
\end{array}\right]=\left[\begin{array}{ll}
\lambda_{\mathrm{I}} & 0
\end{array}\right]\left[\begin{array}{c}
x_{\mathrm{I}} \\
x_{\mathrm{D}}
\end{array}\right],} \\
{\left[\begin{array}{ll}
\mathbf{M}_{\mathrm{DI}} & \mathbf{M}_{\mathrm{DD}}
\end{array}\right]\left[\begin{array}{c}
x_{\mathrm{I}} \\
x_{\mathrm{D}}
\end{array}\right]=\left[\begin{array}{ll}
0 & 0
\end{array}\right]\left[\begin{array}{c}
x_{\mathrm{I}} \\
x_{\mathrm{D}}
\end{array}\right] .}
\end{gathered}
$$

The second part, Eq. (73), is homogeneous, meaning that it does not contribute to the solution (all solutions are trivial-zeros). A key point is that the first part, Eq. (72), which contains all the information on the dynamics of the system is equivalent to the eigenvalue problem of the operator used in the first procedure with conservation conditions:

$$
\frac{\mathrm{d} \Delta \boldsymbol{c}_{\mathrm{I}}}{\mathrm{d} t}=\left[\begin{array}{ll}
\mathbf{M}_{\mathrm{II}} & \mathbf{M}_{\mathrm{ID}}
\end{array}\right]\left[\begin{array}{c}
\Delta \boldsymbol{c}_{\mathrm{I}} \\
\Delta \boldsymbol{c}_{\mathrm{D}}
\end{array}\right] .
$$

Equation (74) is equivalent to Eq. (27). Conservation conditions have been used in this case to replace all the $\Delta c_{\mathrm{D}}$ in this equation. Although not mathematically exact, this simple reasoning may explain the results obtained and be a basis for the conclusion that both procedures should always give the same result.

\section{CONCLUSION}

In this work, two different SNA methods are applied to the reaction network for the oscillatory carbonylation of PEGA as an example of a reaction system with conservation constraints. In both cases, we got the same expressions for alpha coefficients and came to the same conclusion that the basic steady state can be unstable in this system and, as a result, oscillations may occur. It is important to note that the selection of independent variables did not change the result. Thus, in the case under consideration, any combination of three concentrations out of the five intermediate species $\mathrm{PdI}_{2}$, $\mathrm{Pd}, \mathrm{HI}, \mathrm{I}_{2}$, and IPdR gives the same conclusion for stability analysis. In general, the selection of independent variables does not change the result.

The generality of the finding that both mathematical procedures applied to the same model give equal results is further tested using three additional examples. The examples selected include reversible classical substrate-inhibition enzyme mechanism, a complex cell cycle reaction system, and oscillatory intracellular calcium transfer across the cell membrane. In all examples studied, both SNA procedures gave the same result. Since the second procedure is considerably simpler, we would like to propose it to other scientists 
who are dealing with reaction systems having conservation constraints.

\section{ACKNOWLEDGMENTS}

K.N. would like to acknowledge UK Engineering and Physical Sciences Research Council (EPSRC) (Grant No. EP/N033655/1) for financial support. Support from the Ministry of Education, Science and Technological Development of the Republic of Serbia (Grant Nos. 172015 and 45001) is gratefully acknowledged by Ž.Č., S.M., S.A., and Lj. K.-A. Thanks to Dr. Julie Parker for proofreading this article.

\section{APPENDIX: STEADY STATE ANALYSIS}

For the rate constants given in Ref. 5, we found four steady states in a wide interval of reactant concentrations $c_{6}$ as the control parameter (Fig. 1). Four solutions are calculated from the roots of the polynomial:

$$
\begin{aligned}
2 z^{4} & +b\left(K_{1}-a\right) z^{3}-\left[\left(K_{1}-a\right)\left(K_{2}-2 a\right)+b c\right] z^{2} \\
& +c\left(K_{1}+K_{2}-3 a\right) z-c^{2}=0,
\end{aligned}
$$

where $z=c_{2} c_{4} \cdot y=2 c_{4}-c_{2}$ is then calculated as

$$
y=K_{2}-K_{1}-a-b z .
$$

It was then easy to calculate

$$
\begin{aligned}
& c_{4}=\frac{1}{4}\left(y+\sqrt{y^{2}+8 z^{2}}\right), \\
& c_{2}=2 c_{4}-y, \\
& c_{1}=a, \\
& c_{3}=b \sqrt{c_{2} c_{4}}, \\
& c_{5}=\frac{c}{\sqrt{c_{2} c_{4}}} .
\end{aligned}
$$

Parameters used in calculations are

$$
a=\frac{k_{2}}{k_{1} c_{6}}, \quad b=\sqrt{\frac{k_{3}+k_{4} a}{k_{2}}}, \quad c=\frac{k_{5}}{k_{6}} \frac{a}{b}
$$

and

$$
\begin{aligned}
& K_{1}=c_{1}+c_{2}+c_{5}=c_{\mathrm{tot}}(\mathrm{Pd}), \\
& K_{2}=2 c_{1}+c_{3}+2 c_{4}+c_{5}=c_{\mathrm{tot}}(\mathrm{I}) .
\end{aligned}
$$

Two of the steady states given in Fig. 1 are unstable for the whole interval of $c_{6}$ values tested. Unstable steady states are corresponding to lower steady state $c_{3}$ values. Numerical simulation starting in the vicinity of unstable steady states leads to a sudden jump and shift to a stable steady state

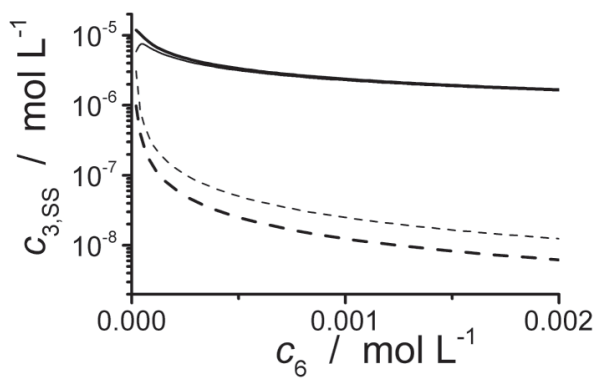

FIG. 1. Steady state concentration $c_{3}$ as a function of concentration of reactant $c_{6}$. Rate constants from Ref. 5 were used.
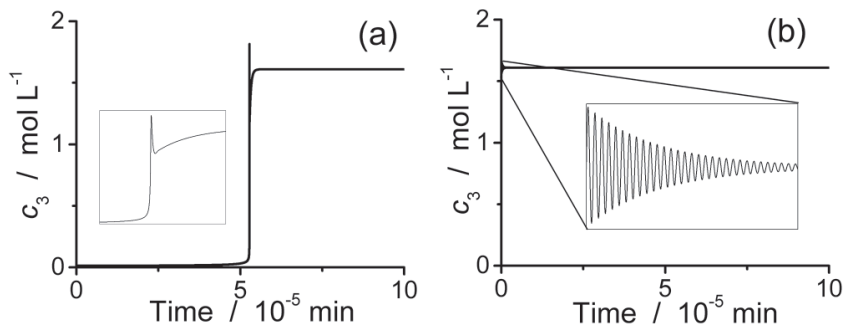

FIG. 2. Numerical simulation of concentration $c_{3}$ evolution from initial points in the vicinity of (a) unstable and (b) stable steady states. Insets are enlarged parts of the diagram with interesting dynamics.

[Fig. 2(a)], while simulation starting in the vicinity of the stable steady state [Fig. 2(b)] leads to damped oscillations. The behaviour is consistent with a saddle node bifurcation of the steady state.

${ }^{1}$ B. L. Clarke, in Advances in Chemical Physics, edited by I. Prigogine and S. Rice (Wiley, New York, 1980), Vol. 43, pp. 1-215.

${ }^{2}$ B. L. Clarke, Cell Biophys. 12, 237 (1988).

${ }^{3}$ J. Parker and K. Novakovic, React. Kinet. Mech. Catal. 118, 73 (2016).

${ }^{4}$ A. Isakova and K. Novakovic, Eur. Polym. J. 95, 430 (2017).

${ }^{5}$ L. Donlon and K. Novakovic, Chem. Commun. 50, 15506 (2014).

${ }^{6}$ D. Hochberg, R. D. B. García, J. A. Á. Bastidas, and J. M. Ribó, Phys. Chem. Chem. Phys. 19, 17618 (2017).

${ }^{7}$ K. Novakovic, C. Grosjean, S. K. Scott, A. Whiting, M. J. Willis, and A. R. Wright, Chem. Phys. Lett. 435, 142 (2007).

${ }^{8}$ K. Novakovic, A. Mukherjee, M. Willis, A. Wright, and S. Scott, Phys. Chem. Chem. Phys. 11, 9044 (2009).

${ }^{9}$ K. Novakovic and J. Parker, Int. J. Chem. Eng. 2011, 1 (2011).

${ }^{10}$ J. Parker and K. Novakovic, Ind. Eng. Chem. Res. 52, 2520 (2013).

${ }^{11}$ L. Donlon, J. Parker, and K. Novakovic, React. Kinet. Mech. Catal. 112, 1 (2014).

${ }^{12}$ J. Parker and K. Novakovic, Eur. J. Chem. Phys. Phys. Chem. 18, 1981 (2017).

${ }^{13}$ J. Parker and K. Novakovic, React. Kinet. Mech. Catal. 123, 113 (2018).

${ }^{14}$ I. R. Epstein and J. A. Pojman, Chaos 9, 255 (1999).

${ }^{15}$ R. P. Washington, W. W. West, G. P. Misra, and J. A. Pojman, J. Am. Chem. Soc. 121, 7373 (1999).

${ }^{16}$ E. Jee, T. Bánsági, A. F. Taylor, and J. A. Pojman, Angew. Chem. Int. Ed. 55, 2127 (2016).

${ }^{17}$ Ž. Čupić, V. Marković, A. Ivanović, and Lj. Kolar-Anić, in Mathematical Modelling, edited by C. R. Brennan (Nova Science Publishers Inc., 2011), pp. 111-178.

${ }^{18}$ Ž. Čupić, G. Schmitz, and Lj. Kolar-Anić, Sci. Publ. State Univ. Novi Pazar Ser. Appl. Math. Inform. Mech. 8, 43 (2016).

${ }^{19}$ V. M. Marković, Ž. Čupić, S. Maćešić, A. Stanojević, V. Vukojević, and Lj. Kolar-Anić, Math. Med. Biol. 33, 1 (2016).

${ }^{20}$ Lj. Kolar-Anić, S. Anić, A. Ivanović-Šašić, N. Pejić, S. Blagojević, and V. Vukojević, in Encyclopaedia of Physical Organic Chemistry, edited by Z. Wang, U. Wille, and E. Juaristi (John Wiley \& Sons, Inc, Hoboken, NJ, 2017), pp. 1127-1222.

${ }^{21}$ Lj. Kolar-Anić and G. Schmitz, J. Chem. Soc. Faraday Trans. 88, 2343 (1992).

${ }^{22}$ Lj. Kolar-Anić, Ž. Čupić, S. Anić, and G. Schmitz, J. Chem. Soc. Faraday Trans. 93, 2147 (1997).

${ }^{23}$ M. Domijan and M. Kirkilionis, J. Math. Biol. 59, 467 (2009).

${ }^{24}$ S. R. Maćešić, Ž. D. Čupić, S. M. Blagojević, N. D. Pejić, S. R. Anić, and Lj. Z. Kolar-Anić, Open Chem. 13, 591 (2015).

${ }^{25}$ P. E. Lehner and E. Noma, Psychometrika 45, 135 (1980).

${ }^{26}$ R. Schuster and S. Schuster, Comput. Appl. Biosci. 9, 79 (1993).

${ }^{27}$ B. L. Clarke and W. Jiang, J. Chem. Phys. 99, 4464 (1993).

${ }^{28}$ V. M. Marković, Ž. Čupić, A. Ivanović, and Lj. Kolar-Anić, Russ. J. Phys. Chem. A 85, 2327 (2011)

${ }^{29}$ G. Schmitz, Lj. Z. Kolar-Anić, S. R. Anić, and Ž. D. Čupić, J. Phys. Chem. A 112, 13452 (2008).

${ }^{30}$ Lj. Kolar-Anić, Ž. Čupić, G. Schmitz, and S. Anić, Chem. Eng. Sci. 65, 3718 (2010).

${ }^{31}$ V. M. Marković, Ž. Čupić, V. Vukojević, and Lj. Kolar-Anić, Endocr. J. 58, 889 (2011). 
${ }^{32}$ S. Maćešić, Ž. Čupić, and Lj. Kolar-Anić, Hem. Ind. 66, 637 (2012).

${ }^{33}$ S. Maćešić, Ž. Čupić, S. Anić, and Lj. Kolar-Anić, Int. J. Non-Linear Mech. 73, 25 (2015).

${ }^{34}$ S. Maćešić, Ž. Čupić, and Lj. Kolar-Anić, React. Kinet. Mech. Catal. 118, 39 (2016).
${ }^{35}$ K. Gaternmann, M. Eiswirth, and A. Sensse, J. Symb. Comput. 40, 1361 (2005).

${ }^{36}$ B. D. Aguda and B. L. Clarke, J. Chem. Phys. 87, 3461 (1987).

${ }^{37}$ B. M. Slepchenko and M. Terasaki, Mol. Biol. Cell 14, 4695 (2003). 